it is to be noted that tam occurs after both numbers whereas mini and mila only after the second. tam is here the distributive suffix whose origin in ta-am we have traced. ${ }^{1}$ )

$$
E Y-M \text { gal-zu. }
$$

The word gal-zu occurring in proper names which has received many explanations and generally adnitted to be of unknown Kassite origin has been discussed again by Hilprecht in $\mathrm{BE} X \mathrm{XX}$ p. 18, where this scholar inclines to read rabut-zu. The phrase occurs in I R. pl. 3 no. $10,1.3$ šag ha-šaggi gal-zu $=$ he who knows well the secret of prayers. As a compound verb in Stele de Vultures obv. 18, 1 gal-na-ga-mu-zu=I am truly very wise. So often in Gudéa Cyl. $\mathrm{B}$, cf. 2,8 gal-mu-zu $=$ he was very wise. The proper names lugal-gal-zu, kur-gal-zu ete. mean ,the king is very sage", "the god-Kuris very wise" etc. Cf. Leipziger Sem. Studien I 2 p. 19.

\section{Miscellen zur Geschichte der bab-ass. Literatur.}

Von Otto Weber.

\section{1) Zum Gilgamesch-Epos.}

In OLZ. 1907 Sp. 10 habe ich für die Stelle Gilgameschepos Tafel VI, Z. 10 die Lesung vorgeschlagen:

lu-še-iș-bit-ka isunarkabtu abnu uknî u hurâsi ,ich (Istar) will dich ergreifen lassen den Wagen von Lasurstein und Gold".

Diese Auffassung der Stelle erhält besondere Bedeutung durch den - mir damals nicht gegenwärtigen - Nachweis Strecks (OLZ. $1905 \mathrm{Sp}$. 376f.), dass das "Ergreifen der Zügel der Istar" sachlich eine assyrische Parallele zu der babylonischen Zeremonie des Ergreifens der Hände Marduks darstelle. In beiden Fallen ist die Redensart eine religiöse Formel für den legitimen Antritt der Königsherrschaft und besagt im Gleichnis dasselbe, was die von mir a. a. Ort bereits herangezogene Parallelstelle aus Nerigal und Erischkigal (Jensen KB. VI 1, S. 78 Z. 17) in kopkreter Form ausdrückt:

1) The article on tam, kam and am was revised, with the help of Hilprechts remarkable introduction to the study of Babylonian mathematics in $B E X X$ after the MS had been sent to the redaction. ,ich will dich ergreifen lassen die Königsherrschaft in der weiten Erde."

Der Wagen, den Gilgamesch ergreifen soll, ist also der Wagen der Istar und der Inhalt des Versprechens ist die Übertragung der Weltherrschaft, was ja auch im Epos selbst unmittelbar darnach (Z. 16 f.) näher ausgeführt wird.

Für die Überlieferungsgeschichte des Epos ist es von Wichtigkeit, dass hier eine spezifisch assyrische Formulierung der Thronbesteigungszeremonie durchschimmert. Nan wird daraus wohl schliessen dürfen, dass die vorliegende Rezension dieser Episode ihre Gestalt erst in verbältnismässig später Zeit erhalten hat. Da aber meines Wissens die Ergreifung der Ziigel der Istar nur ein einziges Mal (K. 2674) und zwar in einer Inschrift Assurbanipals, der auch sonst Zitate aus der Literatur liebt, vorkommt, so wäre auch die Annahme nicht unbedingt abzuweisen, dass in dieser Assurbanipal-Stelle eine Anspielung auf die Episode im Epos vorliegt. Ich halte das gleichwohl für unwahrscheinlich, da der Zusammenhang dieser assyrischen Zeremonie mit dem Akitu-Fest bei Assurbanipal ebenso eng erscheint, wie der der entsprechenden babylonischen. Zudem ist es von vornherein einleuchtender, dass das Epos sich in der Darstellung am passenden Ort an staatsrechtliche und religiöse Bräuche und Zeremonien anlehnt, als dass umgekehit letztere im Epos ihr Vorbild gehabt hätten.

Es ist wohl auch nicht zufällig, dass die eigenartige Einkleidung der von Istar gemachten Versprechungen sich unmittelbar nach der Schilderung des Humbabakampfes findet. Dieser ist in mehr als einer Hinsicht ein Seitenstück zum Tihamatkampf. Im Estherbuch tritt der Gegensatz zwischen Mardochai und Haman (Marduk und Humman. Humban-Humbaba) deutlich hervor (vgl. Zimmern, KAT. ${ }^{3}$ S. 517) und trägt die Züge des Jahresmythus, der auch den Hintergrund des Marduk-Tihamatkampfes bildet. Wie Marduk als Siegespreis die Weltherrschaft zuerkannt wird, so gebührt diese auch dem Humbabatöter Gilgamesch. Dieser Gedankengang hat sicherlich auch den Redaktor des Gilgameschepos bestimmt, die Verheissungen der Istar in die Formel zu kleiden, die für die Übernahme der Königsherrschaft in Assyrien charakteristisch ist.

Die Art und Weise, wie diese Züge des Mythus im Epos novellistisch verflacht und verwischt worden sind, verstärlzt den schon 
aus den obigen Erwägungen gewonnenen Eindruck, dass wir wenigstens diese Episode nur in einer ziemlich späten Rezension besitzen.

Wenu nun durch diese Untersuchungen die Wahrscheinlichkeit, dass der GilgameschHumbaba-Kampf eine Dublette zum MardukTihamat-Kampt ist, noch grösser geworden ist, als sie seither schon war, so werden wir nun auch mit grösserem Recht als bisher daraus Schlüsse für die Komposition des Epos ziehen dürfen. Vielleicht war der eigentliche Kern und die letzte erreichbare Gestalt der Gilgameschsage (nicht des Epos!) eine lokale, erechitische Formulierung des Weltschöpfungsmythus, dessen Hauptageure Gilgamesch-Marduck und Humbaba-Tihamat waren. Allzuweit kann diese Formulierung kaum zurïckreichen, da sie schon durch die Angleichung an zweifellos geschichtliche Erinnerungen (Elamiterkämpfe) den Mythus auf die Stufe der Sage hebt und so bewusstermassen Zeitgeschichte und Urgeschichte $\mathrm{zu}$ sammenrückt, die erstere mit den Zügen der letzteren umkleidet und nach deren Schema erzählt. Dass diese Formulierung aber älter ist als die Komposition des Gilgamesche pos, dass nicht dieses - vielleicht unwillkürlich die Darstellung des Humbabakampfes mit den Zügen des Weltschöpfungsmythus ausgestattet hat, sondern sich an die vorhandene Weltschöpfungssage angelehnt hat, halte ich für zweifellos, auch in dem Falle, dass sich die Auffassung der am Eingang erwähnten Stelle als spezifisch assyrischen Anschauungen Rechnung tragend nicht bewährt. Es bleibt ja immerhin noch die Möglichkeit offen, ist allerdings bis jetzt nicht zu erweisen, dass die fragliche staatsrechtlich-religiöse Zeremonie schon in der alten Istarstadt Erech geübt wurde. Alt und jung sind hier natiir lich ganz allgemeine Begriffe, die nur relative Bedeutung haben. Nirgends muss man vorsichtiger mit ihnen operieren als in der bab.-assyrischen Literatur.

\section{Das Alter der babylonischen Heldensage.}

In wie hobes Alter Gestaltungen mythologischer Stoffe zur Heldensage zurückreichen können, beweist $z$. B. die Legende von dem Könige von Kutha, in der wir den zur Sage gewordenen Schöptungsmythus der Nergalstadt zu erblicken haben. Wir haben sie teilweise in einer Niederschrift aus altbabylonischer Zeit. Für das Alter des Stoffes selbst haben wir einen Anhaltspunkt, wenu sich Hommels Vermutung bestätigt, dass der Text von dem Könige Anubanini von Lulubi (Scheil, Recueil XIV S. $100 \mathrm{ff}$, etwa aus der Zeit des alten Sargon stammend) hier. hergehört. Für das Alter der mythologischen Einkleidung des Stoffes aber hat ebenfalls Hommel ${ }^{1)}$ einen höchst beachtenswerten Fingerzeig gegeben durch den Hinweis auf Gudea, Cyl. A. 25, $27 \mathrm{ff}$, wo in ,den sieben Helden" nach ihm die 7 königlichen Brüder in der Formulierung unserer Legende wieder zu erkennen seien. Dass in diesem ganzen Passus Anspielungen auf historische und mythologische Ereignisse bzw. Figuren zu erkennen sind. ist kaum zweifelhaft. Hommel weist hin auf den unmittelbar vorhergehenden "Helden, der tötet den 6köpfigen Steinbock" und erinnert an 4, R. $30 \mathrm{nr}$. 1. Z. 11, wo der atûdeu Sag VI ,im Bergland getötet wird," Kingi Sag VI = Tamutbal (K 2837) und Gi-in-sag VI ki (Sm. 29). - Dazu füge ich, dass wir in dem ,Babbat (genannt) sag-alim-ma" vielleicht den Tihamat-Töter Marduk (vgl. dessen Namen am Anfang der 7. Tafel), in dem "Löwen, dem Schrecken der Götter" $(26,7-8)$ vielleicht den Lab-bu wiedererkennen dürfen, von dem es doch wohl auch in "Bel und der Lab-bu" (Z. 14) heisst: sich]."

die Götter des Himmels, sie alle [fürchteten

Vgl. auch Jensen, Gilgamesch I, S. 57. Ist das richtig, dann ist damit natïrlich auch die Lesung labbu and die Übersetzung "Löwe" (bei Guden steht Ur-Mah) definitiv sicher gestellt.

Mit diesen Andeutungen mass ich mich heute begnügen. Vielleicht gehen andere Fachgenossen, die solchen. Untersuchungen ausschliesslicher ihre Zeit widmen können, als es mir vergönnt ist, diesen Spuren weiter nach. Nur mit Hilfe solcher Anspielungen auf epische Texte in der übrigen, chronologisch bestimmbaren Literatur dürfen wir hoffen, Anbaltspunkte für die Chronologie der epischen Dichtungen zu gewinnen. Wenn auch da vieles zunächst lediglich Vermutung bleibt, so lohnt es sich doch, einmal den Anfang zu machen, auch auf die Gefahr hin, gelegentlich daneben zu greifen.

Neuburg a. Donau.

1) Brieflich mit Bezugnahme auf meine Darstellung in meiner "Literatur" S. 205, ohne Kenntzis dieser Ausfübrangen. 